SUPPORTING INFORMATION

\title{
A Series of Nonpolar Thymine Analogues of Increasing Size: DNA Base Pairing and Stacking Properties
}

Tae Woo Kim and Eric T. Kool*

Department of Chemistry, Stanford University, Stanford CA 94305-5080

*to whom correspondence should be addressed: kool@stanford.edu

\section{CONTENTS:}

Figure S1. Thermal denaturation plots for stacking studies p. S2

Figure S2. Thermal denaturation plots for thymidine analogue self-pairs $\quad \ldots \quad$ p. S3

Table S1. Measured $\log P$ and calculated physical properties p. S4

Table S2. Thermodynamic data for pairs with natural bases p. S5

Table S3. Thermodynamic data for self-pairs of thymine analogues $\quad$...............S6

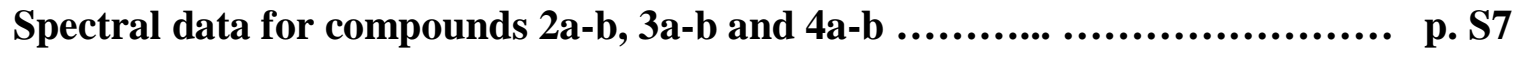

${ }^{1} \mathrm{H}$ NMR spectra for the compounds na and $\mathrm{nb}(\mathrm{n}=1,2,3,4) \ldots \ldots \ldots . . . . . . . . . . . . . . . \quad$ p. S10

Figure S3. HPLC chromatograms of oligonucleotides with H, F, L, B, and I ... p. S18

Table S4. MALDI MS data of oligonucleotides with H, F, L, B, and I $\ldots . . . . \quad$ p. S20

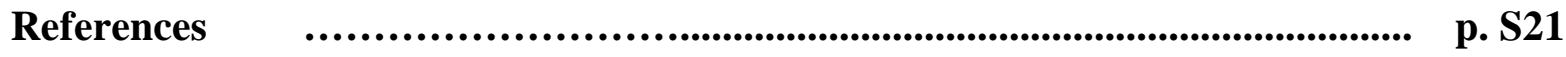


(A)

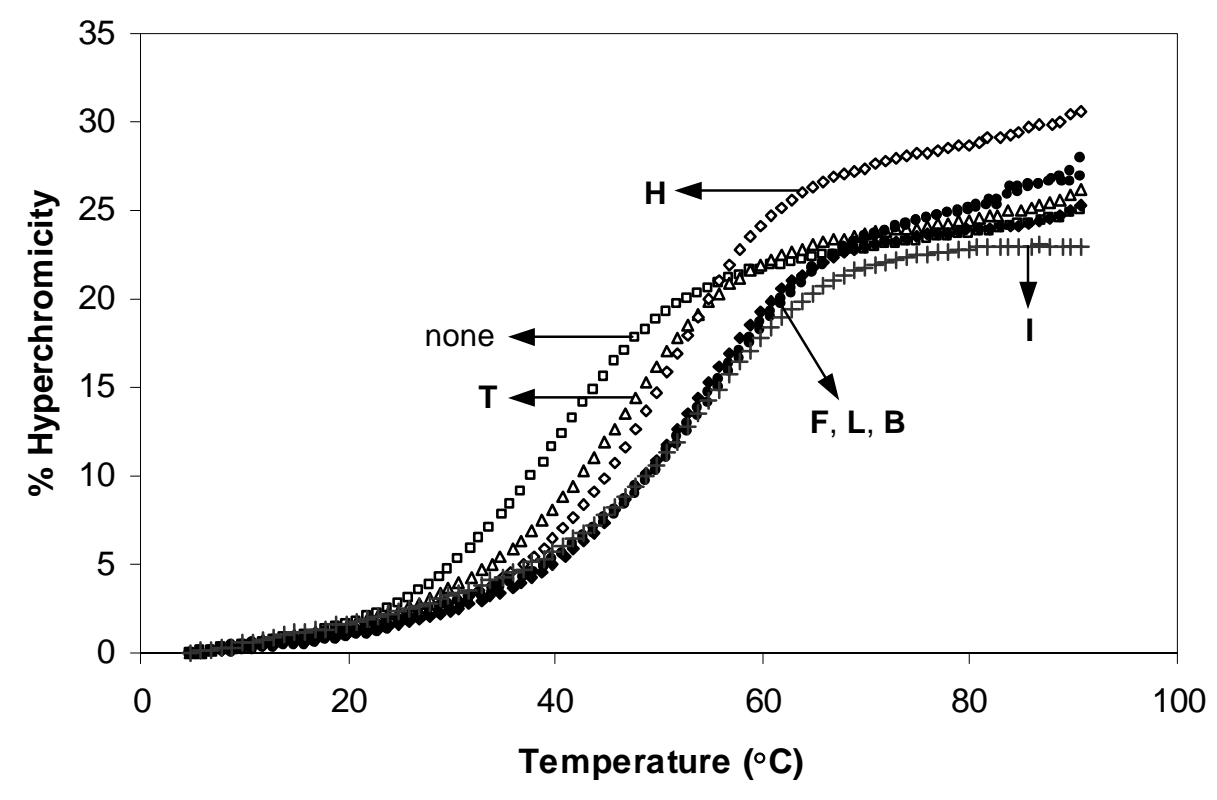

(B)

- none

$\diamond T$

$\Delta \mathrm{H}$

$\square \mathrm{F}$

$\Delta \mathrm{L}$

- B

$*$ I

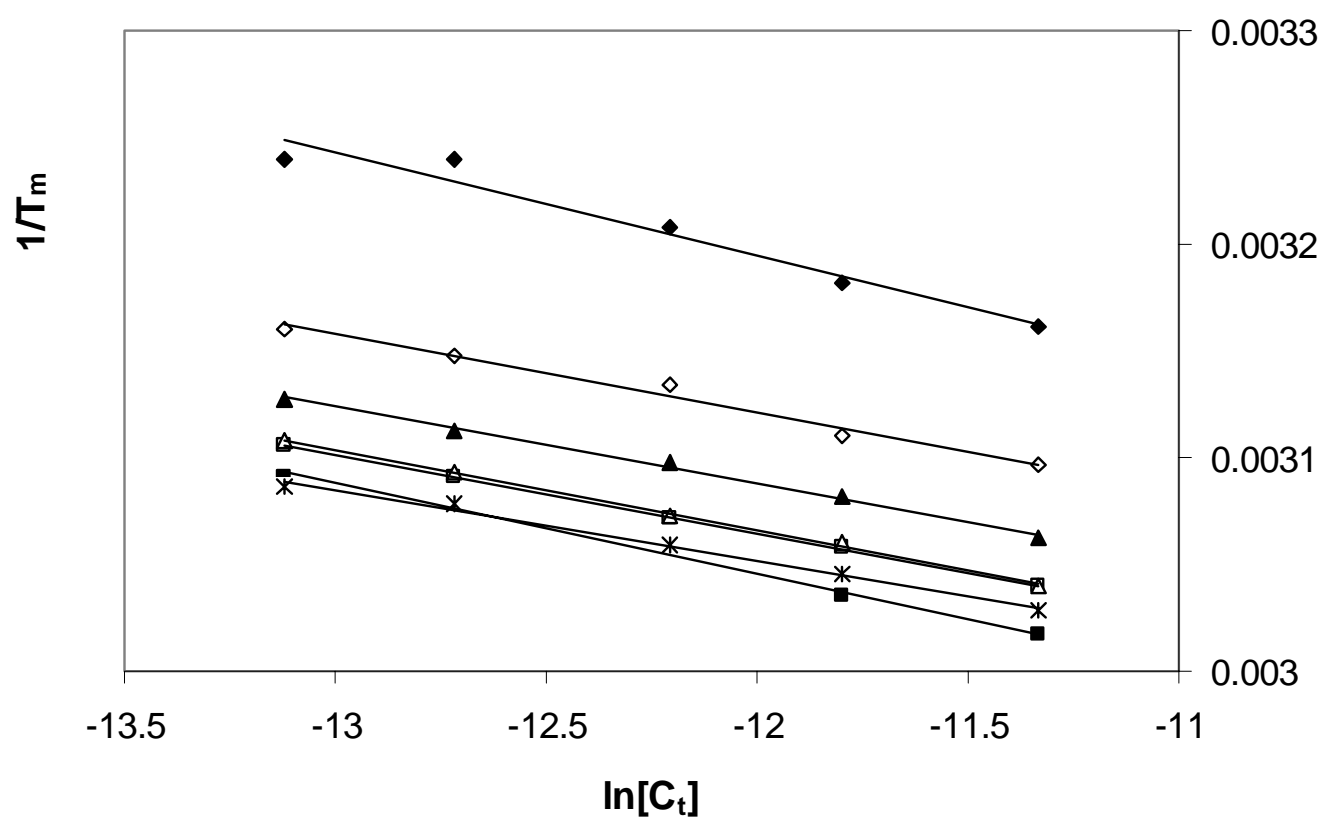

Figure S1. Plots of thermal denaturation data for thymine analogues $(X=\mathbf{H}, \mathbf{F}, \mathbf{L}, \mathbf{B}, \mathbf{I})$ in the 5 ' dangling end position in self-complementary strands (5'-XCGCGCG). (A) Representative melting curves at $5 \mu \mathrm{M}$. (B) Van't Hoff fits for data for the concentration range from 2 to $12 \mu \mathrm{M}$. The buffer contained $1 \mathrm{M} \mathrm{NaCl}, 10 \mathrm{mM}$ phosphate buffer (pH 7.0) with $0.1 \mathrm{mM}$ EDTA. 
(A)

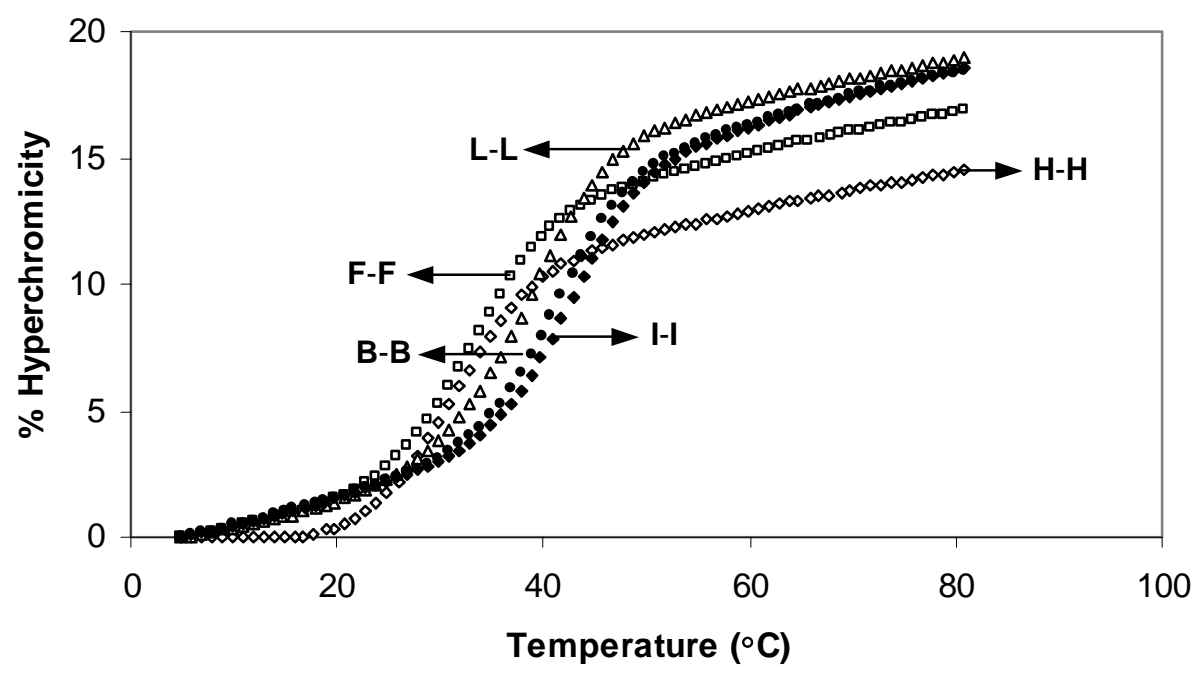

(B)

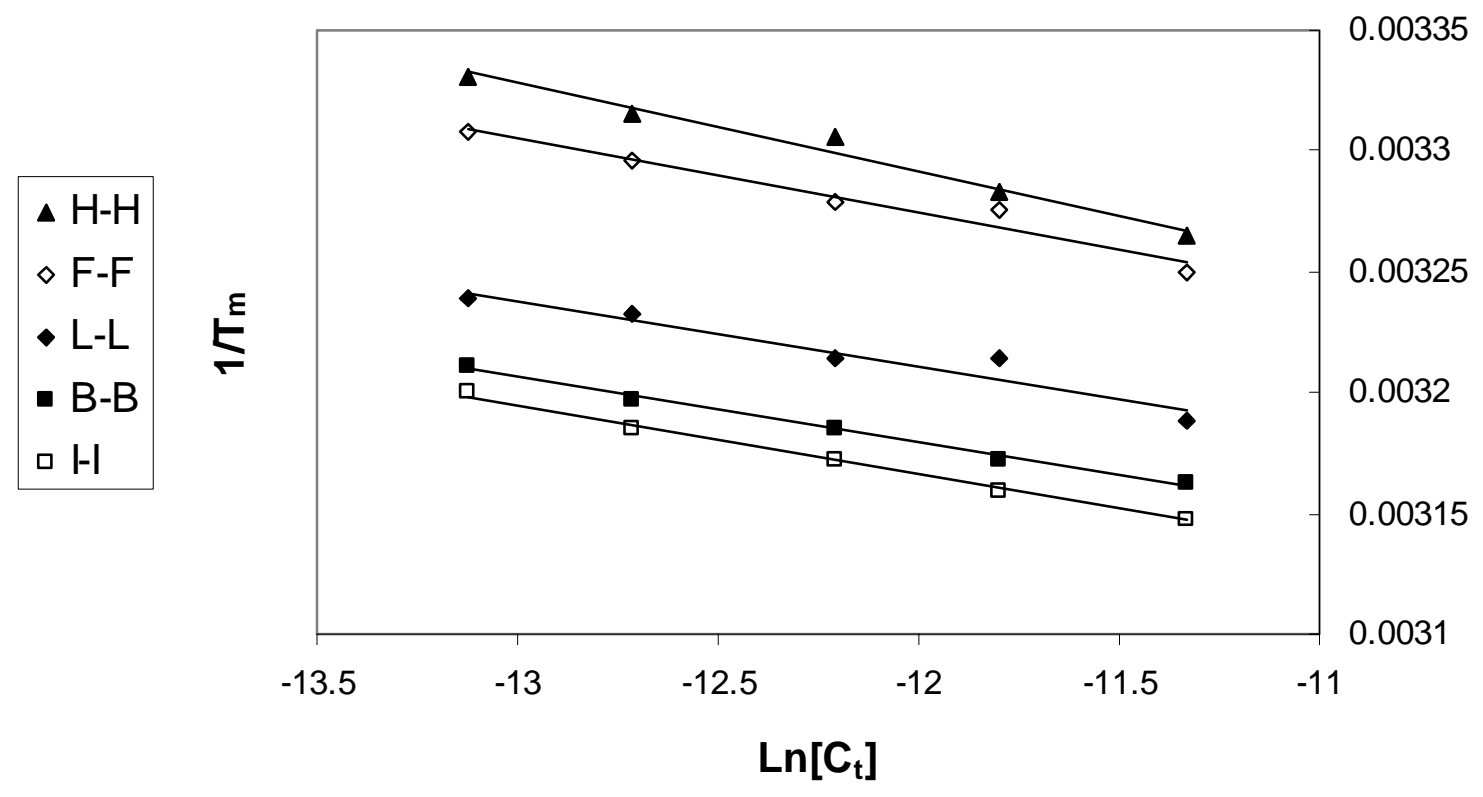

Figure S2. Plots of thermal denaturation data for $12 \mathrm{bp}$ duplexes containing self-pairs of thymine analogues (X = H, F, L, B, I) in a central position. (A) Representative melting curves at $2.5 \mu \mathrm{M}$ for each DNA strand. (B) Van't Hoff fits for data for the concentration range from 1 to $6 \mu \mathrm{M}$. The buffer contained $1 \mathrm{M} \mathrm{NaCl}, 10 \mathrm{mM}$ phosphate buffer ( $\mathrm{pH}$ 7.0) with $0.1 \mathrm{mM}$ EDTA. See Table S2 for sequence and thermodynamic data. 
Table S1. Measured $\log P$ and calculated physical properties of nonpolar thymine analogues ${ }^{\mathrm{a}}$

\begin{tabular}{ccccc}
\hline nucleoside & $\log P($ measured $)$ & $\log P(\text { calculated })^{\mathrm{b}}$ & ${\text { surface area }\left(\AA^{2}\right)^{\mathrm{c}}}^{\text {dipole }(\text { debye })^{\mathrm{c}}}$ \\
\hline $\mathbf{T}$ & $-1.1^{\mathrm{d}}$ & -1.29 & 166 & 4.19 \\
$\mathbf{d H}$ & $0.71^{\mathrm{e}}$ & 1.25 & 154 & 0.26 \\
$\mathbf{d F}$ & $0.78^{\mathrm{e}}$ & 1.57 & 167 & 1.84 \\
$\mathbf{d L}$ & $1.46^{\mathrm{e}}$ & 2.37 & 182 & 1.23 \\
$\mathbf{d B}$ & $2.17^{\mathrm{e}}$ & 2.91 & 190 & 1.31 \\
$\mathbf{d I}$ & $2.30^{\mathrm{e}}$ & 3.97 & 201 & 0.97 \\
\hline
\end{tabular}

${ }^{\mathrm{a}}$ Semi- empirical PM3 calculation (Spartan '02, Wavefunction, Inc.). ${ }^{\mathrm{b}}$ Values are for the Ghose-Crippen method. ${ }^{\mathrm{c}}$ Surface area and dipole moment are for the bases having methyl group at position where it is attached to a nucleoside. ${ }^{\mathrm{d}} \log P($ measured $)=\log \left\{[\mathrm{C}]_{\text {octanol }} /[\mathrm{C}]_{\text {water }}\right\},[\mathrm{C}]_{\text {water }}$ measured, $[\mathrm{C}]_{\text {octanol }}=[\mathrm{C}]_{\text {initial }}-[\mathrm{C}]_{\text {water }},[\mathrm{C}]_{\text {initial }}=0.1$ $\mathrm{mM}$, absorbance measured at $260 \mathrm{~nm} .{ }^{\mathrm{e}} \log P($ measured $)=\log \left\{[\mathrm{C}]_{\text {octanol }} /[\mathrm{C}]_{\text {water }}\right\},[\mathrm{C}]_{\text {octanol }}$ measured, $[\mathrm{C}]_{\text {water }}=$ $[C]_{\text {initial }}-[C]_{\text {octanol }},[C]_{\text {initial }}=0.5 \mathrm{mM}$, absorbance measured at $270 \mathrm{~nm}$ for $\mathbf{d H}, 275 \mathrm{~nm}$ for $\mathbf{d F}$ and $\mathbf{d L}, 285 \mathrm{~nm}$ for $\mathbf{~ d B}$, $295 \mathrm{~nm}$ for dI.

\section{Methods for octanol-water partition coefficients.}

The octanol-water partition coefficients of free nucleosides were determined using the method of Hansch and co-workers. ${ }^{1,2}$ For the partitioning, octanol saturated with distilled water and distilled water saturated with octanol were used. Time-course measurement of octanol saturated with distilled water shows a slow decrease of UV absorbance with time. In order to get reproducible data, freshly prepared, water-saturated octanol was used.

In a typical experiment, a $5.0 \mathrm{~mL}$ portion of a $0.5 \mathrm{mM}$ solution of $\mathbf{d H}$ in water-saturated octanol was shaken with $5.0 \mathrm{~mL}$ of octanol-saturated water in a $15 \mathrm{~mL}$ tube for $5 \mathrm{~min}$. The octanol layer was separated and then centrifuged to remove suspended water. Before and after extraction, the concentration of free nucleoside in octanol solution was determined spectrophotometrically. For negative $\log P$, the water layer was analyzed. 
Table S2. Stabilities of 12 bp DNA duplexes containing a central pair involving a nonpolar thymidine analog $(\mathbf{H}, \mathbf{F}, \mathbf{L}, \mathbf{B}, \mathbf{I})$ paired opposite a natural base $(\mathbf{A}, \mathbf{G}, \mathbf{C}, \mathbf{T})$, as measured by melting temperature $\left(T_{m}\right)$ and free energy $\left(\Delta G^{\circ}{ }_{37}\right)$ for the duplexes.

\begin{tabular}{|c|c|c|c|c|c|}
\hline $\begin{array}{l}\text { Duplex sequence }(\mathrm{X} \bullet \mathrm{Y}) \\
5^{\prime}-\mathrm{CTTTTCXTTCTT} \\
3^{\prime}-\text { GAAAAGYAAGAA }\end{array}$ & $T_{m}\left({ }^{\circ} \mathrm{C}\right)^{\mathrm{a}}$ & $\begin{array}{c}\Delta G_{37}^{\circ} \\
(\mathrm{kcal} / \mathrm{mol})\end{array}$ & $\begin{array}{l}\text { Duplex sequence }(\mathrm{X} \bullet \mathrm{Y}) \\
5^{\prime}-\mathrm{CTTTTCXTTCTT} \\
3^{\prime}-\text { GAAAAGYAAGA }\end{array}$ & $T_{m}\left({ }^{\circ} \mathrm{C}\right)^{\mathrm{a}}$ & $\begin{array}{c}\Delta G_{37}^{\circ} \\
(\mathrm{kcal} / \mathrm{mol})\end{array}$ \\
\hline $\mathrm{T} \bullet \mathrm{A}^{\mathrm{b}}$ & 42.4 & -9.7 & $A \bullet T$ & 42.4 & -9.6 \\
\hline $\mathrm{T} \bullet \mathrm{G}$ & 33.3 & -7.5 & $\mathrm{G} \bullet \mathrm{T}$ & 34.7 & -7.9 \\
\hline $\mathrm{T} \bullet \mathrm{C}$ & 29.5 & -6.6 & $\mathrm{C} \bullet \mathrm{T}$ & 25.7 & -6.0 \\
\hline $\mathrm{T} \bullet \mathrm{T}$ & 29.1 & -6.8 & $\mathrm{~T} \bullet \mathrm{T}$ & 28.8 & -6.8 \\
\hline $\mathrm{H} \bullet \mathrm{A}$ & 23.7 & -5.6 & $\mathrm{~A} \bullet \mathrm{H}$ & 25.3 & -6.2 \\
\hline $\mathrm{H} \bullet \mathrm{G}$ & 23.4 & -5.6 & $\mathrm{G} \bullet \mathrm{H}$ & 24.7 & -6.2 \\
\hline $\mathrm{H} \bullet \mathrm{C}$ & 23.3 & -5.5 & $\mathrm{C} \bullet \mathrm{H}$ & 24.1 & -6.1 \\
\hline $\mathrm{H} \bullet \mathrm{T}$ & 21.8 & -5.5 & $\mathrm{~T} \bullet \mathrm{H}$ & 24.2 & -6.1 \\
\hline$F \bullet A$ & 26.2 & -6.2 & $A \bullet F$ & 27.5 & -6.6 \\
\hline$F \bullet G$ & 23.6 & -6.0 & $G \bullet F$ & 25.1 & -6.4 \\
\hline$F \bullet C$ & 23.7 & -5.8 & $C \cdot F$ & 25.1 & -6.4 \\
\hline $\mathrm{F} \bullet \mathrm{T}$ & 24.0 & -5.9 & $\mathrm{~T} \bullet \mathrm{F}$ & 24.8 & -6.4 \\
\hline $\mathrm{L} \bullet \mathrm{A}$ & 29.8 & -6.9 & $A \cdot L$ & 31.7 & -7.2 \\
\hline $\mathrm{L} \bullet \mathrm{G}$ & 23.9 & -6.6 & $C \cdot \mathrm{L}$ & 24.1 & -5.9 \\
\hline $\mathrm{L} \bullet \mathrm{C}$ & 23.6 & -6.4 & $G \cdot L$ & 24.6 & -6.0 \\
\hline $\mathrm{L} \bullet \mathrm{T}$ & 24.1 & -6.3 & $\mathrm{~T} \bullet \mathrm{L}$ & 25.2 & -6.0 \\
\hline$B \cdot A$ & 27.7 & -6.5 & $A \cdot B$ & 31.5 & -7.1 \\
\hline$B \cdot G$ & 23.4 & -5.9 & $C \cdot B$ & 22.8 & -5.6 \\
\hline$B \cdot C$ & 23.2 & -5.8 & $G \cdot B$ & 24.5 & -5.9 \\
\hline $\mathrm{B} \bullet \mathrm{T}$ & 24.8 & -6.1 & $\mathrm{~T} \bullet \mathrm{B}$ & 26.2 & -6.2 \\
\hline$I \bullet A$ & 28.9 & -6.7 & $A \bullet I$ & 32.1 & -7.3 \\
\hline$I \bullet G$ & 23.9 & -6.1 & $G \bullet I$ & 25.0 & -6.0 \\
\hline$I \bullet C$ & 25.2 & -6.2 & $C \cdot I$ & 23.7 & -5.7 \\
\hline$I \bullet T$ & 26.0 & -6.4 & $T \bullet I$ & 27.6 & -6.3 \\
\hline
\end{tabular}

${ }^{a}$ Conditions: $1 \mathrm{M} \mathrm{NaCl}, 10 \mathrm{mM}$ phosphate buffer (pH 7.0) with $0.1 \mathrm{mM}$ EDTA, $2.5 \mu \mathrm{M}$ each strand, monitored at $260 \mathrm{~nm}$, single concentration - single measurement. Error in $T_{m}$ is estimated at $\pm 0.5^{\circ} \mathrm{C}$, and in free energy, $\pm 10 \%$.

${ }^{\mathrm{b}}$ The standard deviation of six measurements was $\pm 0.1^{\circ} \mathrm{C}$. 
Table S3. Stabilities of 12 bp DNA duplexes containing a central pair composed of nonpolar thymidine analogs $(\mathbf{H}, \mathbf{F}, \mathbf{L}, \mathbf{B}, \mathbf{I})$ paired opposite themselves, as measured by melting temperature $\left(T_{m}\right)$ and free energy $\left(\Delta G^{\circ}{ }_{37}\right)$.

\begin{tabular}{|c|c|c|}
\hline $\begin{array}{l}\text { Duplex sequence }(\mathrm{X} \bullet \mathrm{Y}) \\
5^{\prime}-\mathrm{CTTTTCXTTCTT} \\
3^{\prime}-\text { GAAAAGYAAGA }\end{array}$ & $T_{m}\left({ }^{\circ} \mathrm{C}\right)^{\mathrm{a}}$ & $\begin{array}{c}\Delta G_{37}^{\circ} \\
(\mathrm{kcal} / \mathrm{mol})\end{array}$ \\
\hline $\mathrm{H} \bullet \mathrm{H}$ & 30.4 & -7.0 \\
\hline $\mathrm{F} \bullet \mathrm{H}$ & 29.9 & -6.9 \\
\hline $\mathrm{L} \bullet \mathrm{H}$ & 33.2 & -7.5 \\
\hline $\mathrm{B} \bullet \mathrm{H}$ & 31.1 & -7.2 \\
\hline $\mathrm{I} \bullet \mathrm{H}$ & 31.4 & -7.2 \\
\hline $\mathrm{H} \bullet \mathrm{F}$ & 31.3 & -7.2 \\
\hline$F \bullet F$ & 31.8 & -7.3 \\
\hline $\mathrm{L} \bullet \mathrm{F}$ & 34.2 & -7.8 \\
\hline$B \cdot F$ & 34.9 & -8.0 \\
\hline$I \bullet F$ & 36.1 & -8.2 \\
\hline $\mathrm{H} \bullet \mathrm{L}$ & 32.5 & -7.4 \\
\hline$F \bullet L$ & 34.4 & -7.8 \\
\hline $\mathrm{L} \bullet \mathrm{L}$ & 38.0 & -8.6 \\
\hline$B \cdot L$ & 39.3 & -8.9 \\
\hline$I \bullet L$ & 39.7 & -9.0 \\
\hline $\mathrm{H} \bullet \mathrm{B}$ & 34.1 & -7.7 \\
\hline$F \bullet B$ & 37.2 & -8.4 \\
\hline $\mathrm{L} \bullet \mathrm{B}$ & 41.0 & -9.4 \\
\hline$B \bullet B$ & 41.4 & -9.5 \\
\hline $\mathrm{I} \bullet \mathrm{B}$ & 41.0 & -9.3 \\
\hline $\mathrm{H} \bullet \mathrm{I}$ & 34.5 & -7.8 \\
\hline$F \bullet I$ & 38.2 & -8.7 \\
\hline$L \bullet I$ & 41.8 & -9.6 \\
\hline$B \bullet I$ & 41.6 & -9.5 \\
\hline$I \cdot I^{b}$ & 41.3 & -9.3 \\
\hline
\end{tabular}

${ }^{a}$ Conditions: $1 \mathrm{M} \mathrm{NaCl}, 10 \mathrm{mM}$ phosphate buffer (pH 7.0) with $0.1 \mathrm{mM}$ EDTA, $2.5 \mu \mathrm{M}$ each strand, monitored at $260 \mathrm{~nm}$, single concentration single measurement. Error in $T_{m}$ is estimated at $\pm 0.5^{\circ} \mathrm{C}$, and in free energy, $\pm 10 \%$.

${ }^{\mathrm{b}}$ The standard deviation of six measurements was $\pm 0.1^{\circ} \mathrm{C}$. 
Spectral data $\left({ }^{1} \mathrm{H}\right.$ NMR, ${ }^{13} \mathrm{C}$ NMR, HRMS) for compounds $2 \mathrm{a}-\mathrm{b}, 3 \mathrm{a}-\mathrm{b}$ and $4 \mathrm{a}-\mathrm{b}$ 1',2'-Dideoxy- $\beta$-1'-(2,4-dichloro-5-methylphenyl)-5'-O-(4,4'-dimethoxytrityl)-D-

ribofuranose (2a). ${ }^{1} \mathrm{H} \mathrm{NMR}\left(\mathrm{CDCl}_{3}, \mathrm{ppm}\right) 7.54(\mathrm{~s}, 1 \mathrm{H}), 7.46(\mathrm{~d}, 2 \mathrm{H}, \mathrm{J}=8.0 \mathrm{~Hz}), 7.36-7.33(\mathrm{~m}$, 4H), 7.30-7.20 (m, 4H), $6.83(\mathrm{dd}, 4 \mathrm{H}, \mathrm{J}=9.0,1.5 \mathrm{~Hz}), 5.41(\mathrm{dd}, 1 \mathrm{H}, \mathrm{J}=10,6 \mathrm{~Hz}), 4.41-4.39(\mathrm{~m}$, 1H), 4.07-4.05 (m, 1H), $3.79(\mathrm{~s}, 6 \mathrm{H}), 3.37$ (app d, 2H, J = 4.5 Hz), $2.46(\mathrm{ddd}, 1 \mathrm{H}, \mathrm{J}=14,6.0$, $5.0 \mathrm{~Hz}), 2.22(\mathrm{~s}, 3 \mathrm{H}), 2.04-1.87(\mathrm{~m}, 1 \mathrm{H}) ;{ }^{13} \mathrm{C} \mathrm{NMR}\left(\mathrm{CDCl}_{3}, \mathrm{ppm}\right) 158.4,144.7,138.4,135.9$, $134.9,133.3,130.0,129.0,128.6,128.1,127.8,126.8,113.1,86.3,85.8,76.4,74.4,64.1,55.2$, 42.4, 19.5; HRMS (FAB+, NBA matrix) calcd mass 601.1524 for $\left[\mathrm{C}_{33} \mathrm{H}_{32} \mathrm{Cl}_{2} \mathrm{O}_{5}\right]$, found 601.1553.

\section{1',2'-Dideoxy- $\beta$-1'-(2,4-dichloro-5-methylphenyl)-5'-O-(4,4'-dimethoxytrityl)-D-}

ribofuranose cyanoethyl $\mathrm{N}, \mathrm{N}$-diisopropylphosphoramidite (2b). ${ }^{1} \mathrm{H} \mathrm{NMR}\left(\mathrm{CDCl}_{3}, \mathrm{ppm}\right) 7.62$ (app d, 1H, J = 9.5 Hz), 7.49-7.46 (m, 2H), 7.38-7.32 (m, 4H), 7.29-7.25 (m, 2H), 7.23-7.19 (m, 2H), 6.84-6.80 (m, 4H), 5.40 (app quintet, 1H, J = 5Hz, H1'), 4.52-4.45 (m, 1H, H3'), 4.21 (br, $\left.1 \mathrm{H}, \mathrm{H} 4^{\prime}\right), 3.85-3.75(\mathrm{~m}, 1 \mathrm{H}), 3.78\left(\operatorname{app~d}, 6 \mathrm{H}, \mathrm{J}=3.5 \mathrm{~Hz}, \mathrm{OCH}_{3}\right), 3.68-3.54(\mathrm{~m}, 3 \mathrm{H}), 3.40$ (app ddd, $\left.1 \mathrm{H}, \mathrm{J}=22.10,3.9 \mathrm{~Hz}, \mathrm{H} 5^{\prime}\right), 3.27$ (app t+d, 1H, J = 10.6 (for triplet), 3.7 (for doublet) Hz, $\left.\mathrm{H5}^{\prime}\right), 2.61(\operatorname{app~t}, 1 \mathrm{H}, \mathrm{J}=6.0 \mathrm{~Hz}), 2.63-2.51\left(\mathrm{~m}, 1 \mathrm{H}, \mathrm{H} 2^{\prime}\right), 2.44(\operatorname{app~t}, 1 \mathrm{H}, \mathrm{J}=6.5 \mathrm{~Hz}), 2.22$ $\left(\operatorname{app} \mathrm{d}, 3 \mathrm{H}, \mathrm{J}=5.0 \mathrm{~Hz}, \mathrm{ArCH}_{3}\right)$ 1.90-1.83 (m, 1H, H2'), 1.18-1.15 (m, 8H), $1.06(\operatorname{app} \mathrm{d}, 4 \mathrm{H}, \mathrm{J}=$ $6.5 \mathrm{~Hz}) ;{ }^{13} \mathrm{C} \mathrm{NMR}\left(\mathrm{CDCl}_{3}, \mathrm{ppm}\right)$ 158.7, $145.1(\mathrm{~d}), 138.6$ (d), 136.3 (m), 135.3, 133.6, 130.4 (d), $129.3(d), 129.6$ (d), 128.5 (d), 128.1, 127.1 (d), 117.8 (d), 113.4, 86.5, 85.8 (d), 76.0 (t), 64.0 (d), $58.6(\mathrm{t}), 55.5(\mathrm{~d}), 43.5(\mathrm{t}), 42.1,31.9,24.8(\mathrm{~m}), 22.9,20.6(\mathrm{~d}), 20.4(\mathrm{~d}), 19.9,14.4 ;{ }^{31} \mathrm{P}$ NMR $\left(\mathrm{CDCl}_{3}\right.$, ppm, external $\mathrm{H}_{3} \mathrm{PO}_{4}$ standard) 149.5, 148.7; HRMS (FAB+, NBA matrix) calcd mass 801.2602 for $\left[\mathrm{C}_{42} \mathrm{H}_{49} \mathrm{Cl}_{2} \mathrm{~N}_{2} \mathrm{O}_{6} \mathrm{P}\right]$, found 801.2600. 


\section{$1^{\prime}, 2^{\prime}$-Dideoxy- $\beta$-1'-(2,4-dibromo-5-methylphenyl)-5'-O-(4,4'-dimethoxytrityl)-D-}

ribofuranose (3a). ${ }^{1} \mathrm{H} \mathrm{NMR}\left(\mathrm{CDCl}_{3}, \mathrm{ppm}\right) 7.80(\mathrm{~s}, 1 \mathrm{H}), 7.47(\mathrm{~d}, 2 \mathrm{H}, \mathrm{J}=7.0 \mathrm{~Hz}), 7.38-7.35(\mathrm{~m}$, 4H), $7.30(\operatorname{app~t,~2H,~J~=~8.0~Hz),~} 7.21(\operatorname{app~t,~2H,~J~=~} 7.0 \mathrm{~Hz}), 6.85(\mathrm{~d}, 4 \mathrm{H}, \mathrm{J}=9.0 \mathrm{~Hz}), 5.33(\mathrm{dd}$, $1 \mathrm{H}, \mathrm{J}=9.5,6.0 \mathrm{~Hz}), 4.37-4.34(\mathrm{~m}, 1 \mathrm{H}), 4.06-4.04(\mathrm{~m}, 1 \mathrm{H}), 3.78(\mathrm{~s}, 6 \mathrm{H}), 3.39(\operatorname{app} \mathrm{dd}, 1 \mathrm{H}, \mathrm{J}=10$ $4.5 \mathrm{~Hz}), 3.33(\operatorname{app} \mathrm{dd}, 1 \mathrm{H}, \mathrm{J}=10,5.0 \mathrm{~Hz}), 2.50(\mathrm{ddd}, 1 \mathrm{H}, \mathrm{J}=13.5,6.0,2.5 \mathrm{~Hz}), 2.34(\mathrm{~s}, 3 \mathrm{H})$, 1.87-1.82 (m, 1H); ${ }^{13} \mathrm{C}$ NMR $\left(\mathrm{CDCl}_{3}, \mathrm{ppm}\right) 158.7,145.0,141.0,138.7,136.1,134.3,130.7$, $130.3,128.3,128.1,127.0,124.5,119.9,113.4,86.2,78.6,74.6,64.4,55.5,42.7,22.6$; HRMS (FAB+, NBA matrix) calcd mass 689.0514 for $\left[\mathrm{C}_{33} \mathrm{H}_{32} \mathrm{Br}_{2} \mathrm{O}_{5}\right]$, found 689.0534.

\section{1',2'-Dideoxy- $\beta-1^{\prime}$-(2,4-dibromo-5-methylphenyl)-5'-O-(4,4'-dimethoxytrityl)-D-}

ribofuranose cyanoethyl $\boldsymbol{N}, \boldsymbol{N}$-diisopropylphosphoramidite (3b). ${ }^{1} \mathrm{H} \mathrm{NMR}\left(\mathrm{CDCl}_{3}, \mathrm{ppm}\right) 7.87$ (app d, 1H, J = 5.0 Hz), 7.50-7.47 (m, 2H), 7.40-7.36 (m, 4H), 7.32-7.25 (m, 2H), 7.23-7.19 (m, 2H), 6.87-6.82 (m, 4H), 5.32 (app quintet, 1H, J = 5 Hz, H1'), 4.47-4.40 (m, 1H, H3'), 4.21 (br, $\left.1 \mathrm{H}, \mathrm{H} 4^{\prime}\right), 3.87-3.76(\mathrm{~m}, 1 \mathrm{H}), 3.79\left(\operatorname{app~d}, 6 \mathrm{H}, \mathrm{J}=3.5 \mathrm{~Hz}, \mathrm{OCH}_{3}\right), 3.68-3.52(\mathrm{~m}, 3 \mathrm{H}), 3.39-3.26$ (m, 2H), 2.69-2.55 (m, 1H, H2'), 2.61 (app t+d, 1H, J = 6.5 (for triplet), 1.5 (for doublet) Hz), $2.43(\operatorname{app} \mathrm{t}+\mathrm{d}, 1 \mathrm{H}, \mathrm{J}=6.5$ (for triplet), 1.0 (for doublet) $\mathrm{Hz}), 2.35\left(\mathrm{~s}, 3 \mathrm{H}, \mathrm{ArCH}_{3}\right), 1.84-1.78(\mathrm{~m}$, $\left.1 \mathrm{H}, \mathrm{H} 2^{\prime}\right), 1.18-1.15(\mathrm{~m}, 8 \mathrm{H}), 1.05(\operatorname{app~d}, 4 \mathrm{H}, \mathrm{J}=6.5 \mathrm{~Hz}) ;{ }^{13} \mathrm{C} \mathrm{NMR}\left(\mathrm{CDCl}_{3}, \mathrm{ppm}\right) 158.7(\mathrm{t})$, 145.1 (d), 140.9 (d), 138.8 (d), 136.2 (t), 134.4 (d), 130.8 (d), 130.4 (q), 128.5 (d), 128.1 (d), 127.0 (d), 124.6 (d), 120.0, 117.8 (d), 113.4, 86.5, 86.0, 85.7 (d), 79.0 (d), 75.8 (d), 64.2 (d), 58.6 (m), 55.5 (d), 43.5 (m),42.1, 34.9, 31.9, 25.5, 24.8 (m), 22.8 (d), 20.6 (d), 20.4 (d), 14.4; ${ }^{31} \mathrm{P}$ NMR $\left(\mathrm{CDCl}_{3}\right.$, ppm, external $\mathrm{H}_{3} \mathrm{PO}_{4}$ standard) 149.0, 148.2; HRMS (FAB+, NBA matrix) calcd mass 889.1592 for $\left[\mathrm{C}_{42} \mathrm{H}_{49} \mathrm{Br}_{2} \mathrm{~N}_{2} \mathrm{O}_{6} \mathrm{P}\right]$, found 889.1597 .

\section{1',2'-Dideoxy- $\beta$-1'-(2,4-diiodo-5-methylphenyl)-5'-0-(4,4'-dimethoxytrityl)-D-}

ribofuranose (4a). ${ }^{1} \mathrm{H} \mathrm{NMR}\left(\mathrm{CDCl}_{3}, \mathrm{ppm}\right) 8.00(\mathrm{~s}, 1 \mathrm{H}), 7.64(\mathrm{~s}, 1 \mathrm{H}), 7.48(\mathrm{~d}, 2 \mathrm{H}, \mathrm{J}=7.2 \mathrm{~Hz})$, 
7.39-7.36 (m, 4H), 7.30 (app t, 2H, J = 7.6 Hz), 7.23-7.19 (m, 1H), $6.85(\mathrm{dd}, 4 \mathrm{H}, \mathrm{J}=7.2,2.4 \mathrm{~Hz})$, $5.18(\mathrm{dd}, 1 \mathrm{H}, \mathrm{J}=9.6,6.0 \mathrm{~Hz}), 4.37-4.34(\mathrm{~m}, 1 \mathrm{H}), 4.06-4.03(\mathrm{~m}, 1 \mathrm{H}), 3.79(\mathrm{~s}, 6 \mathrm{H}), 3.37$ (app d, $2 \mathrm{H}, \mathrm{J}=4.4 \mathrm{~Hz}), 2.51(\mathrm{ddd}, 1 \mathrm{H}, \mathrm{J}=13.2,6.0,2.4 \mathrm{~Hz}), 2.35(\mathrm{~s}, 3 \mathrm{H}), 1.85-1.78(\mathrm{~m}, 1 \mathrm{H}) ;{ }^{13} \mathrm{C} \mathrm{NMR}$ $\left(\mathrm{CDCl}_{3}, \mathrm{ppm}\right) 158.7,145.0,143.9,142.6,139.6,136.9,136.1,130.3,128.3,128.1,127.0,113.4$ 101.6, 96.1, 86.6, 86.4, 74.4, 64.3, 55.4, 42.9, 27.4; HRMS (MALDI+, CHCA) calcd mass785.0229 for $\left[\mathrm{C}_{33} \mathrm{H}_{32} \mathrm{I}_{2} \mathrm{O}_{5}+\mathrm{Na}\right]$, found 785.0260.

\section{1',2'-Dideoxy- $\beta$-1'-(2,4-diiodo-5-methylphenyl)-5'-O-(4,4'-dimethoxytrityl)-D-}

ribofuranose cyanoethyl $\boldsymbol{N}, \boldsymbol{N}$-diisopropylphosphoramidite (4b). ${ }^{1} \mathrm{H} \mathrm{NMR}\left(\mathrm{CDCl}_{3}, \mathrm{ppm}\right) 8.08$ (app d, 1H, J = 2.4 Hz), $7.65(\mathrm{~s}, 1 \mathrm{H}), 7.49(\operatorname{app~t}, 2 \mathrm{H}, \mathrm{J}=6.0 \mathrm{~Hz}), 7.41-7.37(\mathrm{~m}, 4 \mathrm{H}), 7.33-7.28$ $(\mathrm{m}, 2 \mathrm{H}), 7.23-7.19(\mathrm{~m}, 1 \mathrm{H}), 6.86(\mathrm{dd}, 4 \mathrm{H}, \mathrm{J}=8.8,6.4 \mathrm{~Hz}), 5.17($ app quintet, $1 \mathrm{H}, \mathrm{J}=4.4 \mathrm{~Hz}$, H1'), 4.48-4.39 (m, 1H), 4.20 (br, 1H), 3.88-3.80 (m, 1H), 3.79 (app d, 6H, J = 2.8 Hz, OCH $)_{3}$, 3.76-3.56 (m, 3H), 3.43-3.35 (m, 1H), 3,29-3.24 (m, 1H), 2.71-2.55 (m, 1H, H2'), 2.62 (app t, 1H, $\mathrm{J}=6.8 \mathrm{~Hz}), 2.43(\operatorname{app~t}, 1 \mathrm{H}, \mathrm{J}=6.4 \mathrm{~Hz}), 2.36\left(\mathrm{~s}, 3 \mathrm{H}, \mathrm{ArCH}_{3}\right), 1.18-1.16(\mathrm{~m}, 8 \mathrm{H}), 1.83-1.73(\mathrm{~m}$, 1H, H2'), 1.05 (app d, 4H, J = 7.2 Hz); ${ }^{13} \mathrm{C} \mathrm{NMR}\left(\mathrm{CDCl}_{3}, \mathrm{ppm}\right)$ 158.7, 145.1 (d), 143.6 (d), 142.7, 139.7, 137.0 (d), 136.2 (q), 130.4 (q), 127.0 (d), 117.7 (d), 113.4, 101.7 (d), 96.2, 86.3 (m), 83.0 (d), 75.8 (d), 64.1 (d), 58.6 (q), 55.5, 43.5 (q), 42.3, 31.8, 27.5, 24.8 (t), 22.9, 20.5 (q); ${ }^{31} \mathrm{P}$ NMR ( $\mathrm{CDCl}_{3}$, ppm, external $\mathrm{H}_{3} \mathrm{PO}_{4}$ standard) 149.1, 148.6; HRMS (FAB+, NBA matrix) calcd mass 962.1405 for $\left[\mathrm{C}_{42} \mathrm{H}_{49} \mathrm{I}_{2} \mathrm{~N}_{2} \mathrm{O}_{6} \mathrm{P}\right]$, found 962.1445 . 


\section{${ }^{1} \mathrm{H}$ NMR spectra of $5^{\prime}$ - $O$-tritylated $\beta$-C-Nucleosides}

$1^{\prime}, 2^{\prime}$-Dideoxy- $\beta$-1'-(3-methylphenyl)-5'-O-(4,4'-dimethoxytrityl)-D-ribofuranose (1a)

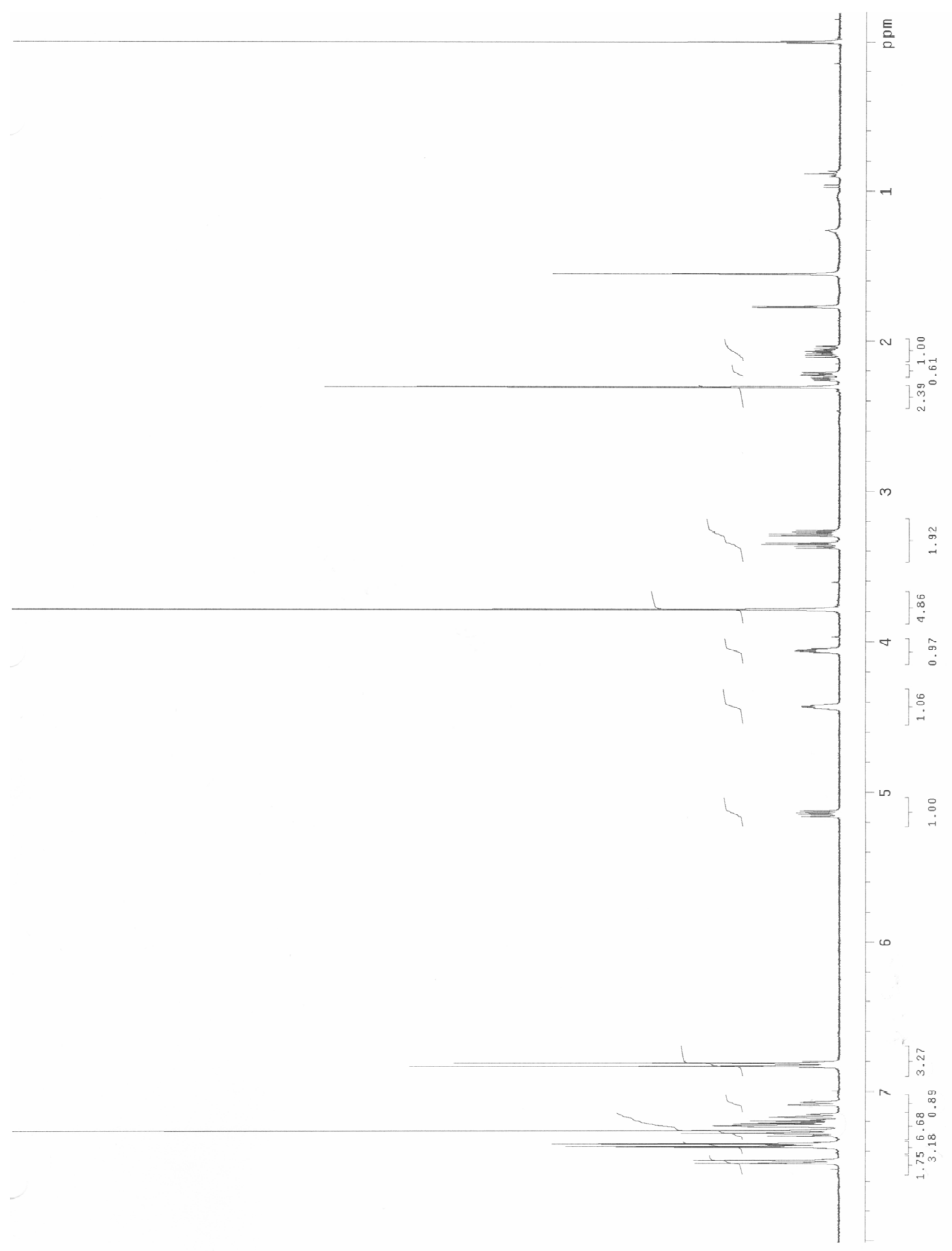


$1^{\prime}, 2^{\prime}$-Dideoxy- $\beta$-1'-(2,4-dichloro-5-methylphenyl)-5'-O-(4,4'-dimethoxytrityl)-D-ribofuranose (2a)

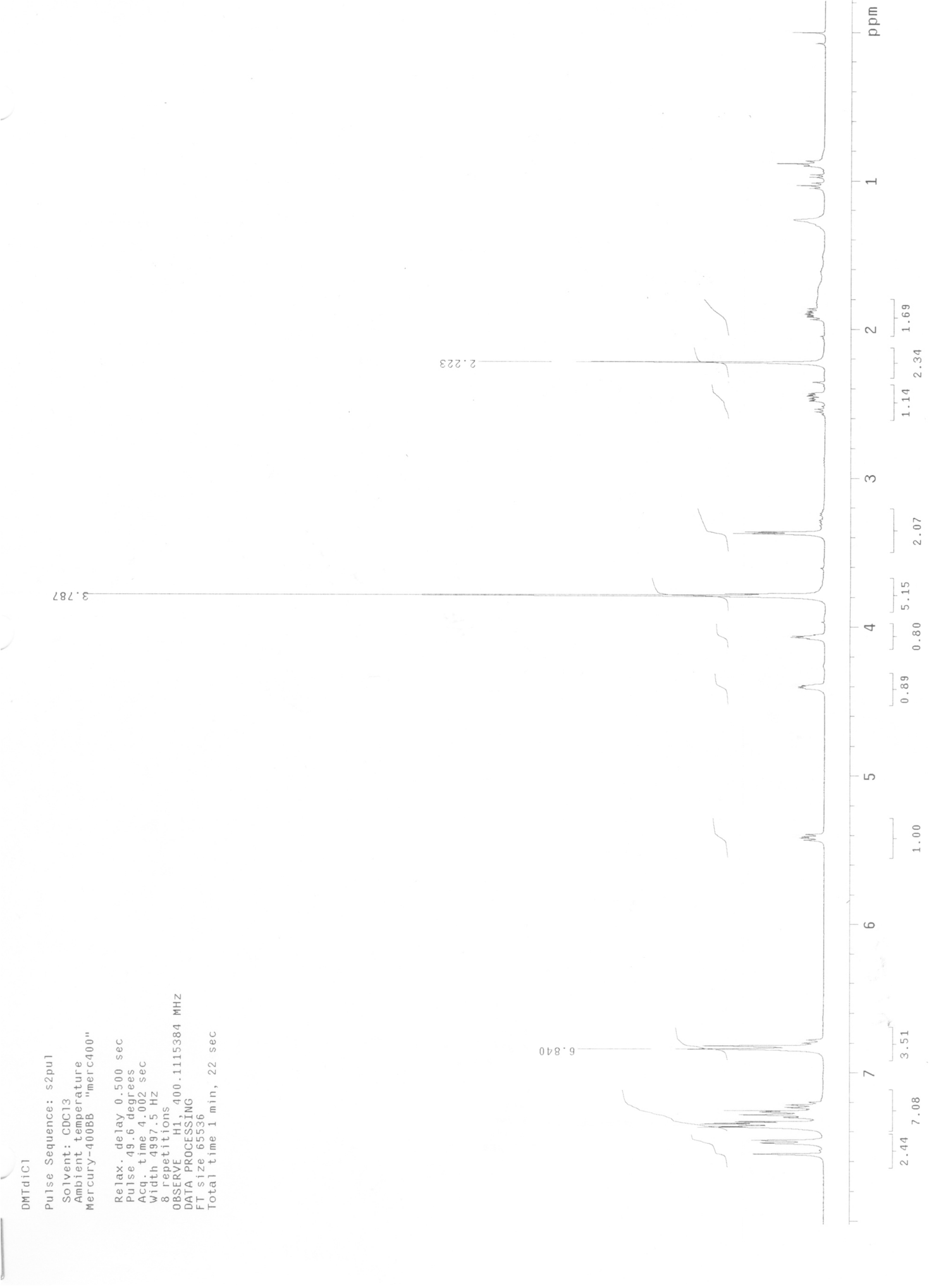


$1^{\prime}, 2^{\prime}$-Dideoxy- $\beta$-1'-(2,4-dibromo-5-methylphenyl)-5'-O-(4,4'-dimethoxytrityl)-D-ribofuranose (3a)

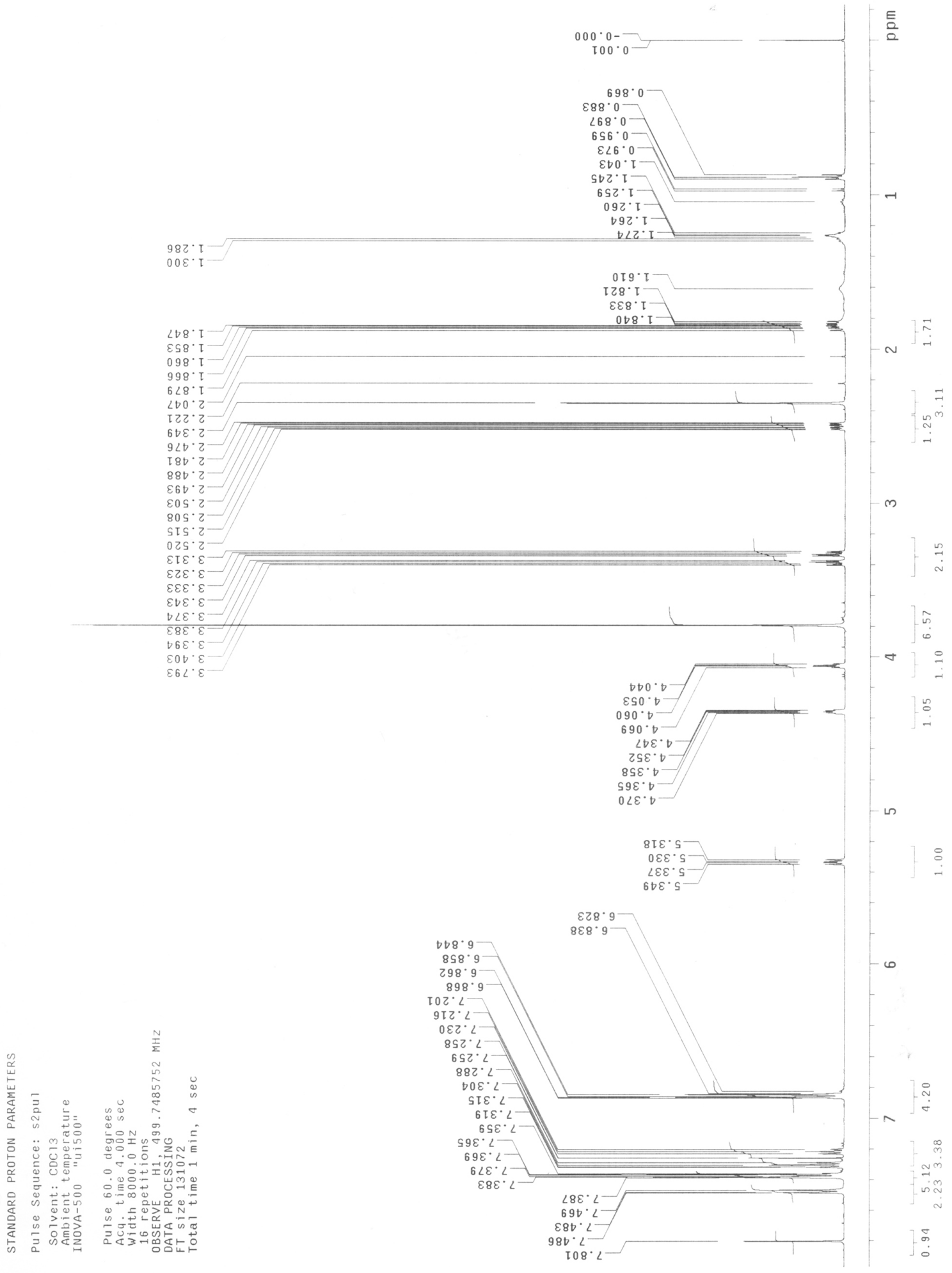


$1^{\prime}, 2^{\prime}$-Dideoxy- $\beta$-1'-(2,4-diiodo-5-methylphenyl)-5'-O-(4,4'-dimethoxytrityl)-D-ribofuranose (4a)

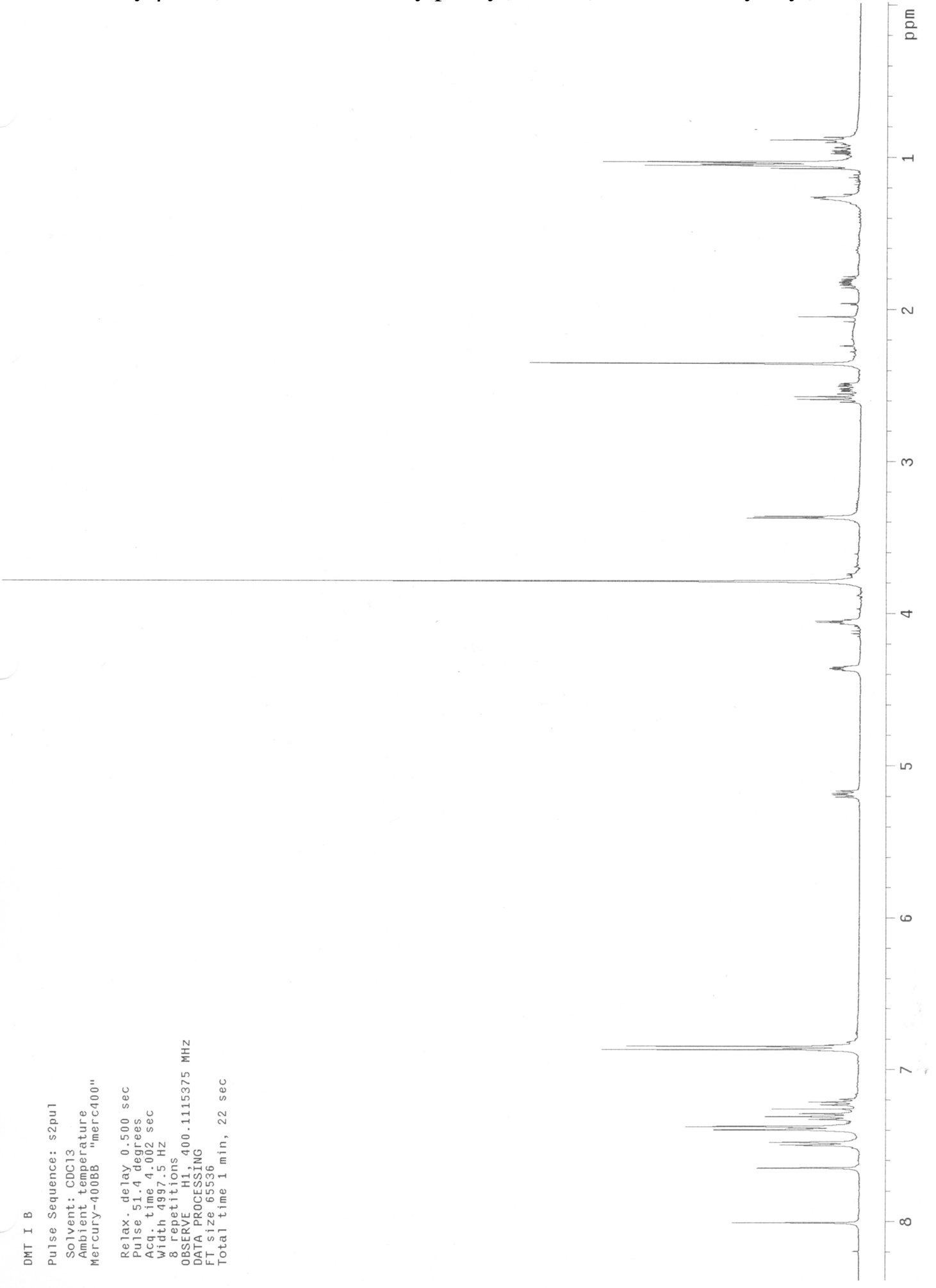




\section{${ }^{1}$ H NMR of 3'-O-Phosphoramidites}

$1^{\prime}, 2^{\prime}$-Dideoxy- $\beta$ - $1^{\prime}$-(3-methylphenyl)-5'-O-(4,4'-dimethoxytrityl)-D-ribofuranose cyanoethyl $N, N$-diisopropylphosphoramidite (1)

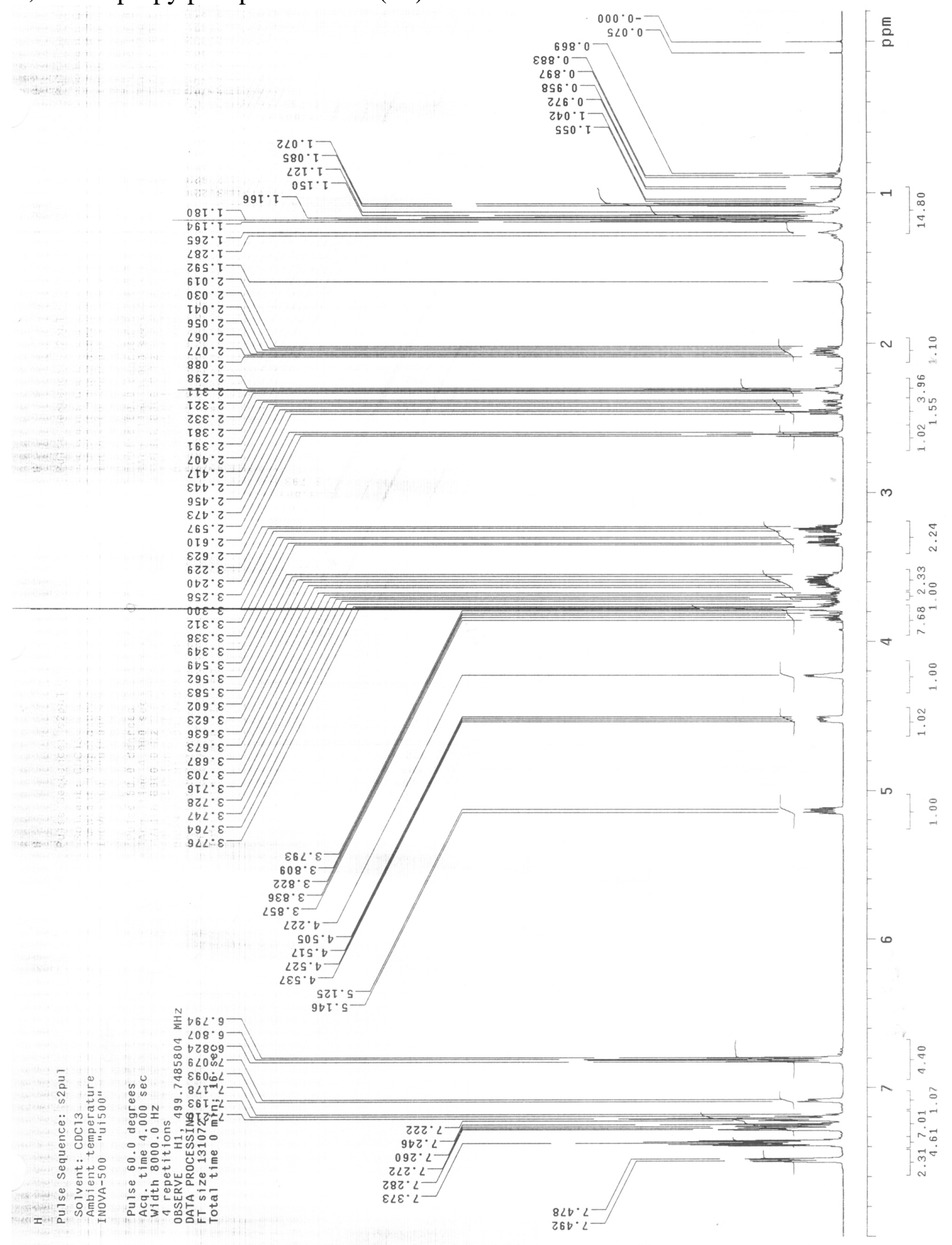


$1^{\prime}, 2^{\prime}$-Dideoxy- $\beta$-1'-(2,4-dichloro-5-methylphenyl)-5'-O-(4,4'-dimethoxytrityl)-D-ribofuranose cyanoethyl $N, N$-diisopropylphosphoramidite (2b)

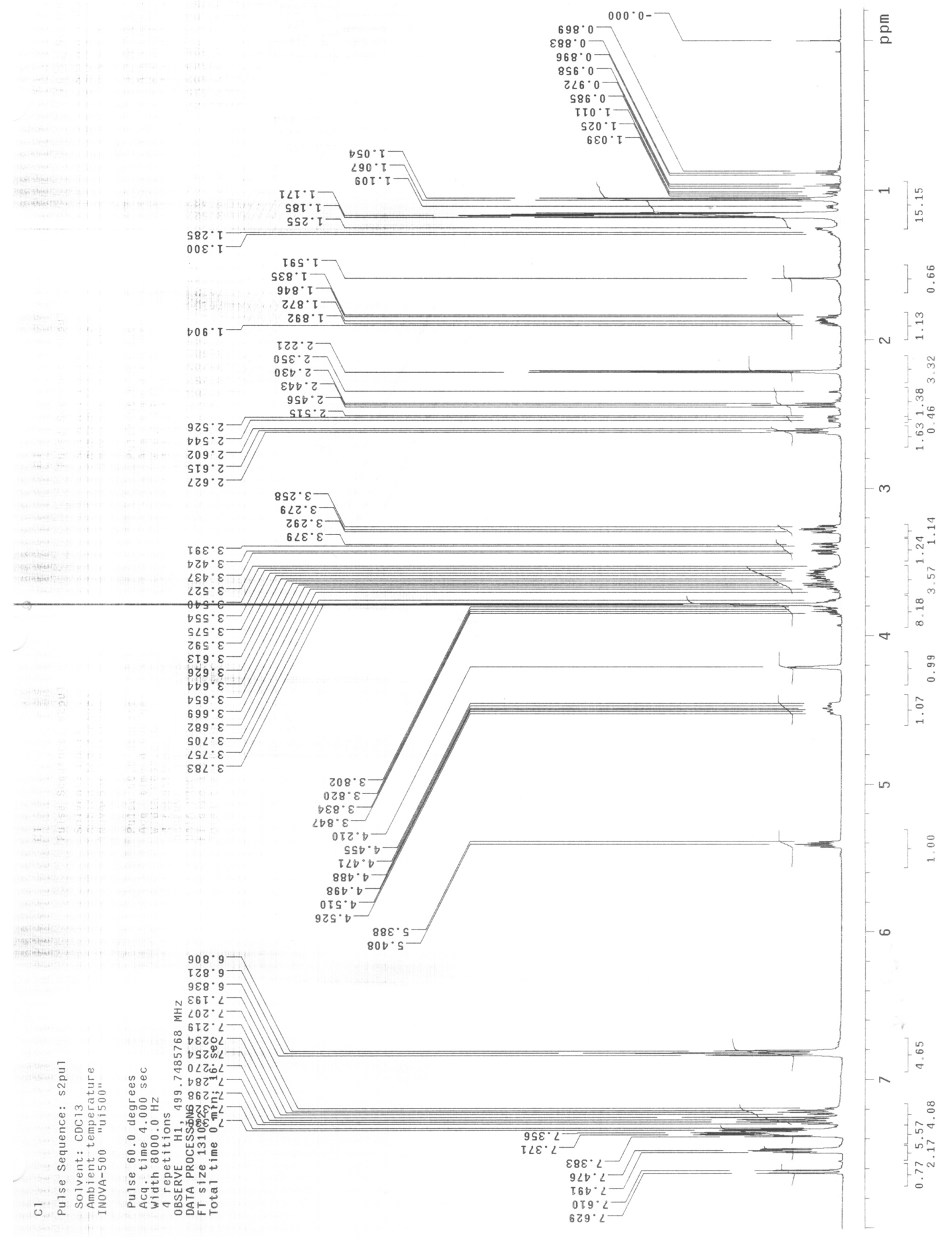


$1^{\prime}, 2^{\prime}$-Dideoxy- $\beta$-1'-(2,4-dibromo-5-methylphenyl)-5'-O-(4,4'-dimethoxytrityl)-D-ribofuranose cyanoethyl $N, N$-diisopropylphosphoramidite (3b)

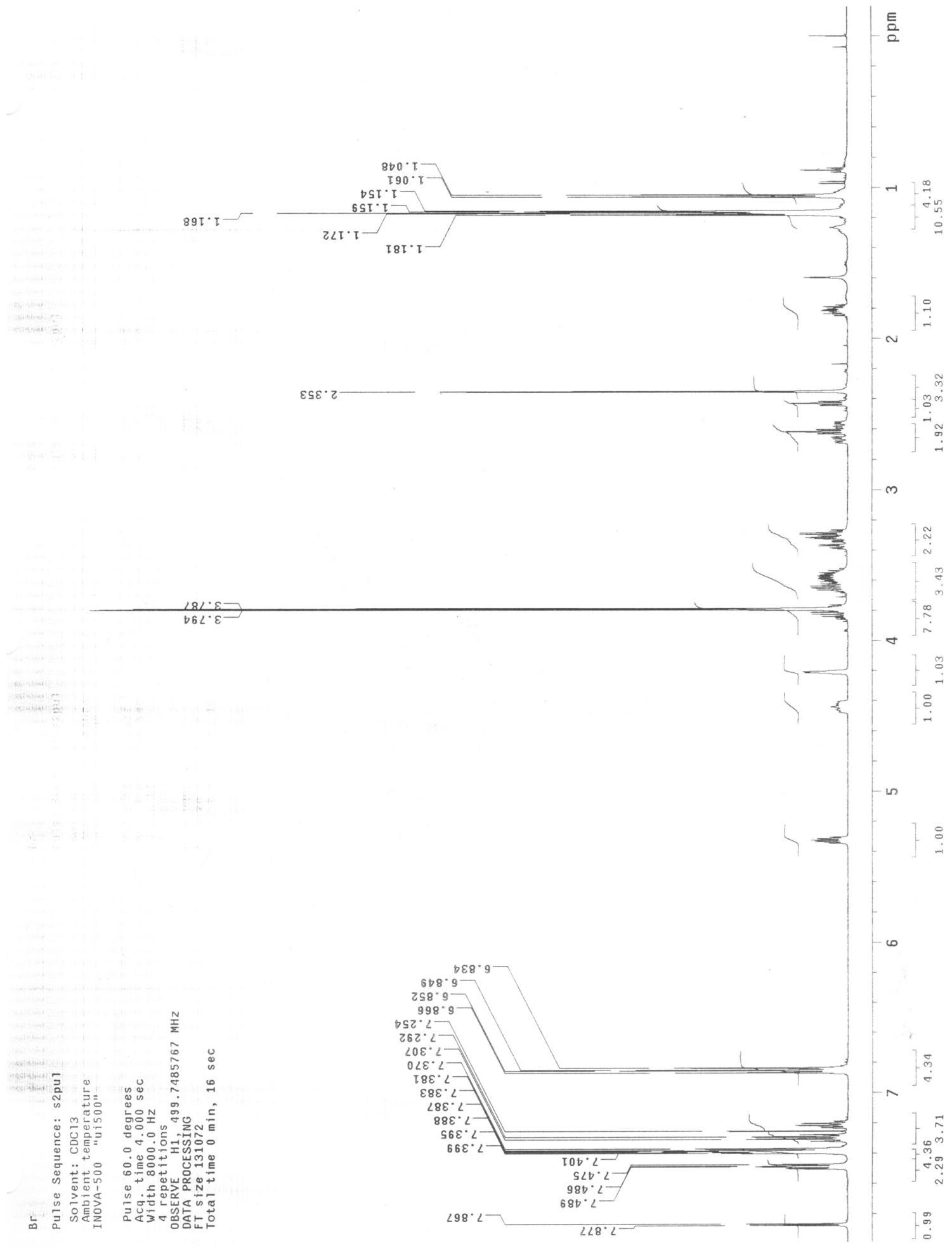


$1^{\prime}, 2^{\prime}$-Dideoxy- $\beta$-1'-(2,4-diiodo-5-methylphenyl)-5'-O-(4,4'-dimethoxytrityl)-D-ribofuranose cyanoethyl $N, N$-diisopropylphosphoramidite (4b)

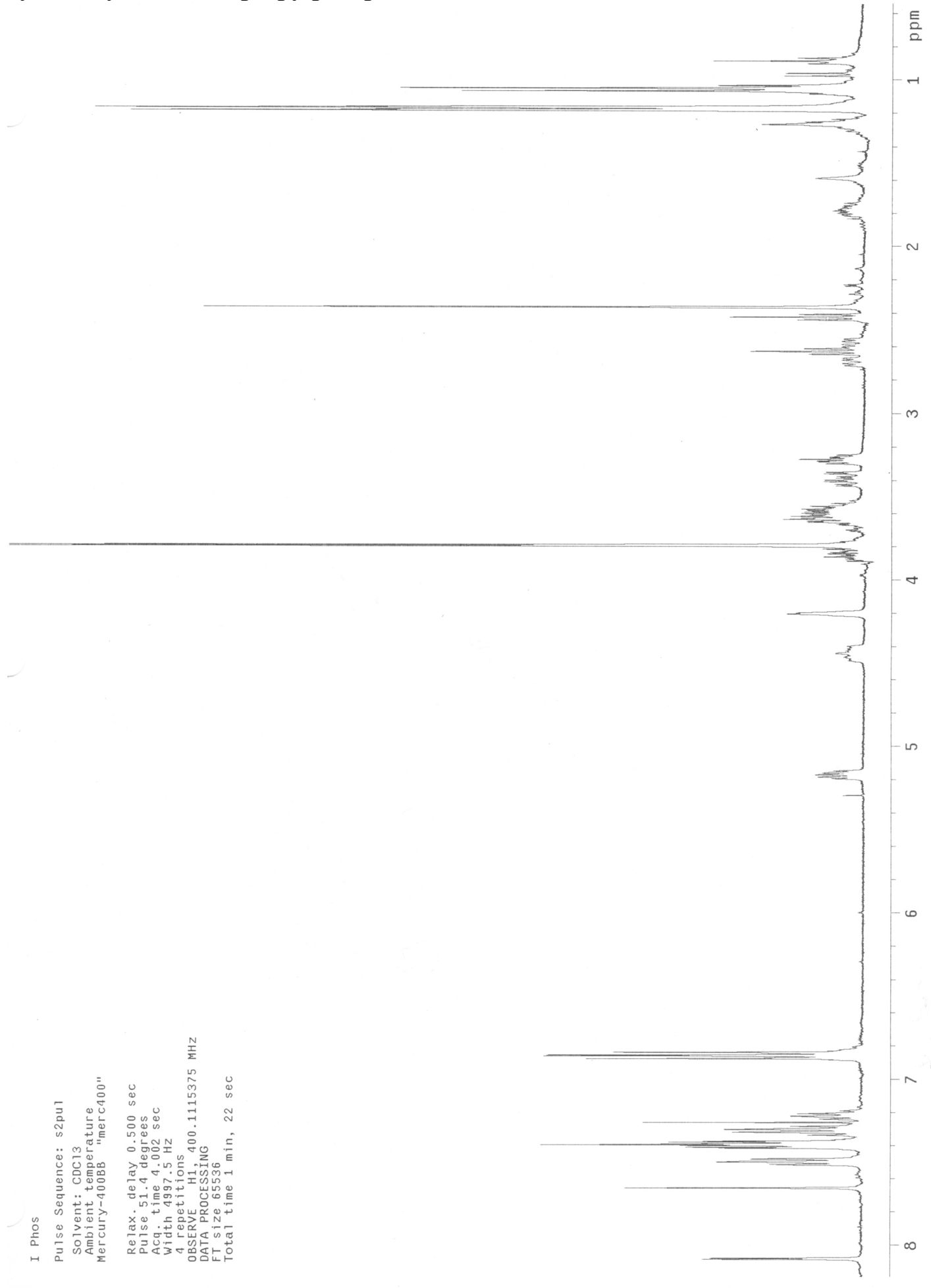


Figure S3. HPLC chromatograms of oligonucleotides with H, F, L, B, and I

A) Base Pairing Oligomers ${ }^{\mathrm{a}}$

5'-CTTTTCHTTCTT (5)

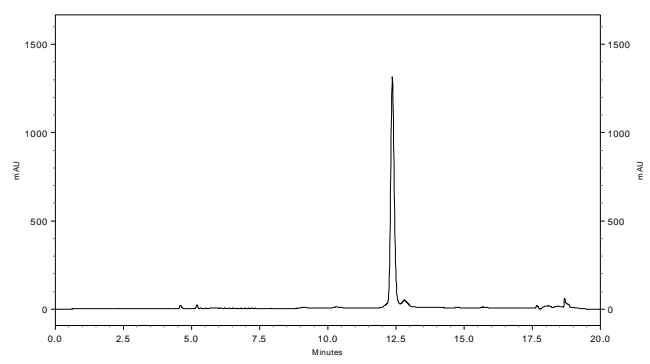

5'-CTTTTCFTTCTT (6)

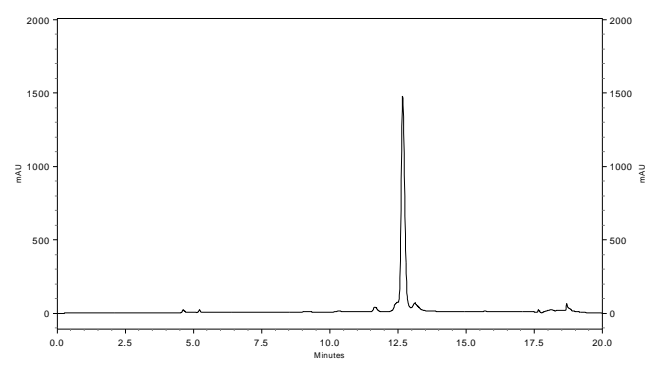

5'-CTTTTCLTTCTT (7)

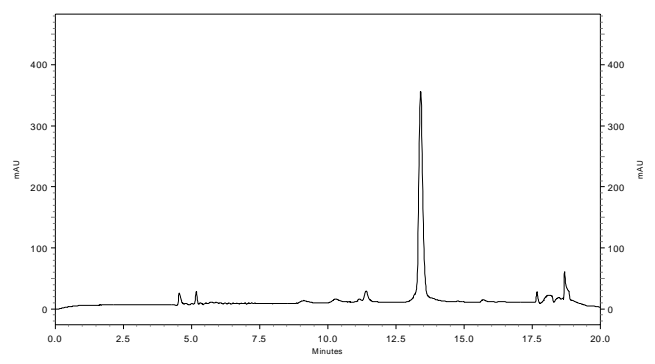

5'-CTTTTCВTTCTT ${ }^{\mathrm{b}}(\mathbf{8})$

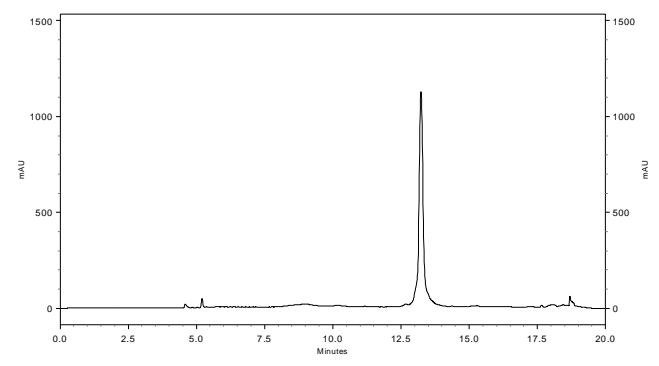

5'-CTTTTCITTCTT ${ }^{\mathrm{b}}(\mathbf{9})$

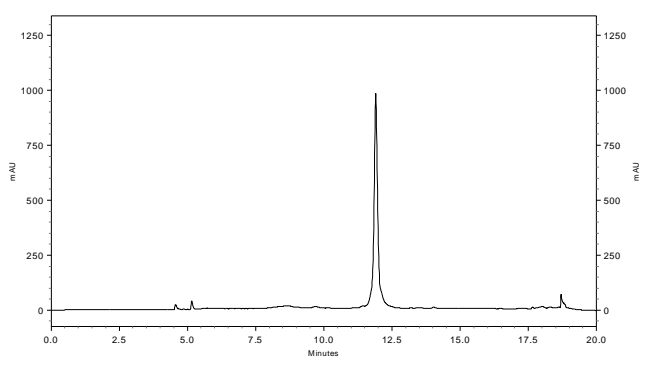

3'-GAAAAGHAAGAA (10)

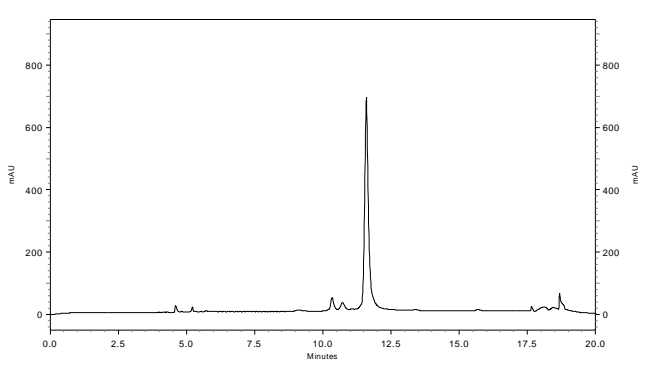

3'-GAAAAGFAAGAA (11)

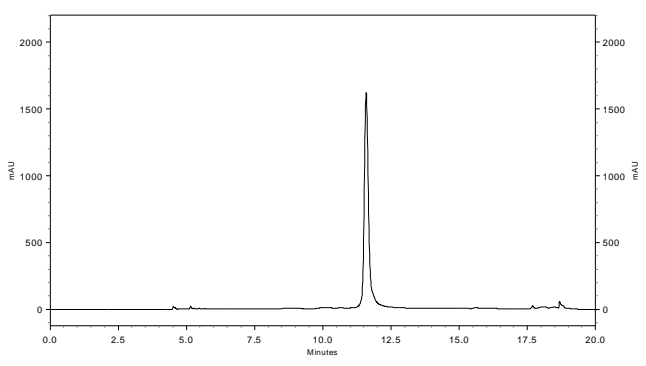

3'-GAAAAGLAAGAA (12)

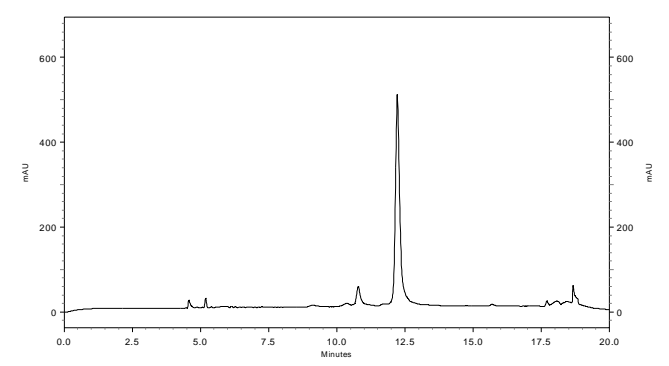


3'-GAAAAGBAAGAA (13)

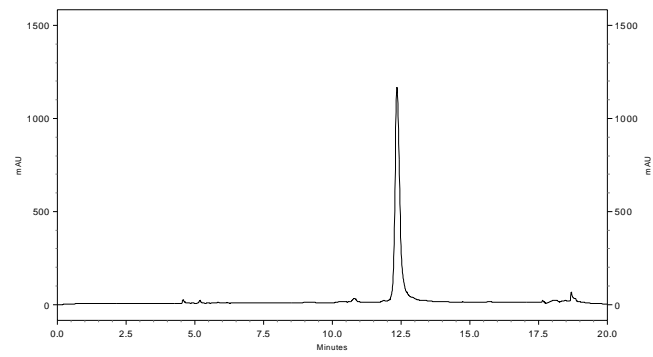

3'-GAAAAGIAAGAA (14)

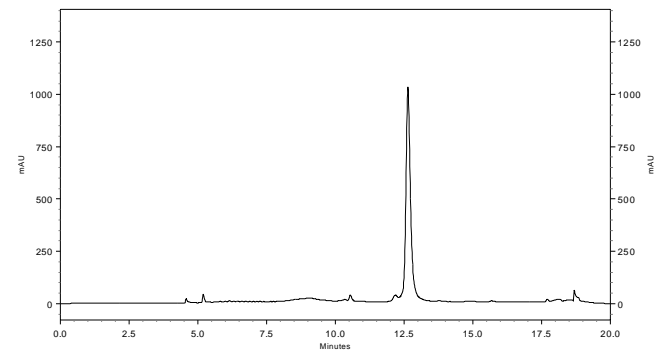

${ }^{a} 0$ to $25 \%, 15$ min gradient $\mathrm{CH}_{3} \mathrm{CN} / 50 \mathrm{mM}$ TEAA.

${ }^{\mathrm{b}} 0$ to $30 \%, 15$ min gradient $\mathrm{CH}_{3} \mathrm{CN} / 50 \mathrm{mM}$ TEAA.

B) Stacking Oligomers ${ }^{a}$

\section{5'-HCGCGCG (15)}

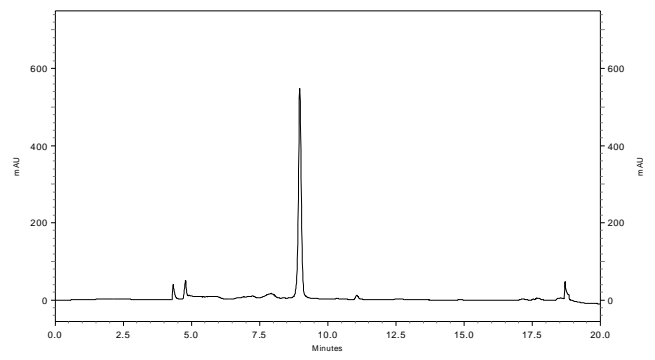

5'-FCGCGCG (16)

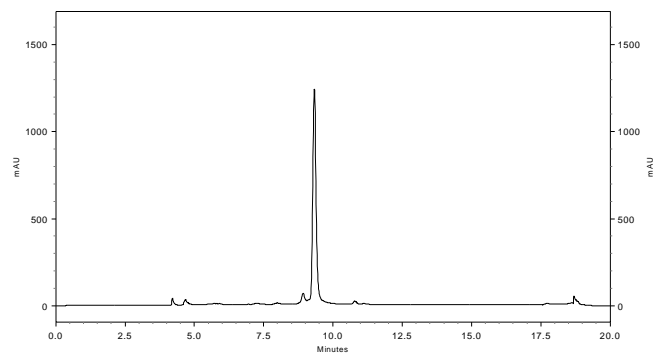

5'-LCGCGCG (17)

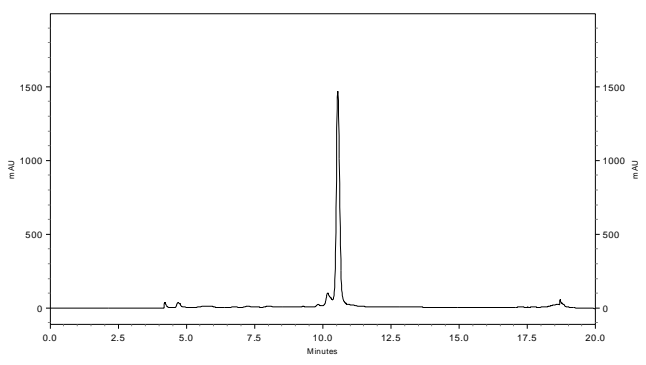

5'-BCGCGCG (18)

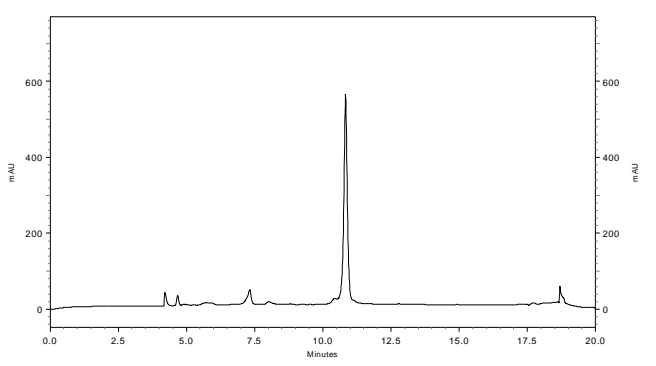

5'-ICGCGCG (19)

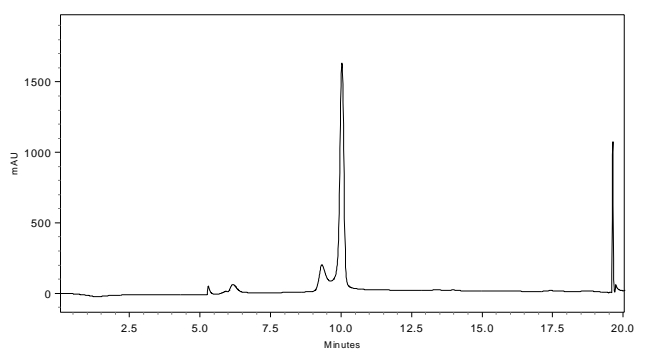

${ }^{\text {a }} 0$ to $40 \%, 15$ min gradient $\mathrm{CH}_{3} \mathrm{CN} / 50 \mathrm{mM}$ TEAA. 
Table S4. MALDI MS data of oligonucleotides with H, F, L, B and I

A) Base Pairing Oligomers ${ }^{\mathrm{a}}$

\begin{tabular}{clcc}
\hline Sequence & & calcd MS & obsd MS \\
\hline 5'-CTTTTCHTTCTT & $\mathrm{C}_{119} \mathrm{H}_{156} \mathrm{~N}_{25} \mathrm{O}_{77} \mathrm{P}_{11}$ & 3508.4 & 3508.2 \\
5'-CTTTTCFTTCTT & $\mathrm{C}_{119} \mathrm{H}_{154} \mathrm{~F}_{2} \mathrm{~N}_{25} \mathrm{O}_{77} \mathrm{P}_{11}$ & 3544.3 & 3543.7 \\
5'-CTTTTCLTTCTT & $\mathrm{C}_{119} \mathrm{H}_{154} \mathrm{Cl}_{2} \mathrm{~N}_{25} \mathrm{O}_{77} \mathrm{P}_{11}$ & 3577.2 & 3577.3 \\
5'-CTTTTCBTTCTT & $\mathrm{C}_{11} \mathrm{H}_{154} \mathrm{Br}_{2} \mathrm{~N}_{25} \mathrm{O}_{77} \mathrm{P}_{11}$ & 3666.1 & 3666.5 \\
5'-CTTTTCITTCTT & $\mathrm{C}_{119} \mathrm{H}_{154} \mathrm{I}_{2} \mathrm{~N}_{25} \mathrm{O}_{77} \mathrm{P}_{11}$ & 3760.1 & 3760.2 \\
3'-GAAAAGHAAGAA & $\mathrm{C}_{122} \mathrm{H}_{148} \mathrm{~N}_{55} \mathrm{O}_{61} \mathrm{P}_{11}$ & 3700.5 & 3701.0 \\
3'-GAAAAGFAAGAA & $\mathrm{C}_{122} \mathrm{H}_{146} \mathrm{~F}_{2} \mathrm{~N}_{55} \mathrm{O}_{61} \mathrm{P}_{11}$ & 3736.5 & 3737.7 \\
3'-GAAAAGLAAGAA & $\mathrm{C}_{122} \mathrm{H}_{146} \mathrm{Cl}_{2} \mathrm{~N}_{55} \mathrm{O}_{61} \mathrm{P}_{11}$ & 3769.4 & 3769.8 \\
3'-GAAAAGBAAGAA & $\mathrm{C}_{122} \mathrm{H}_{146} \mathrm{Br}_{2} \mathrm{~N}_{55} \mathrm{O}_{61} \mathrm{P}_{11}$ & 3858.3 & 3859.1 \\
3'-GAAAAGIAAGAA & $\mathrm{C}_{122} \mathrm{H}_{146} \mathrm{I}_{2} \mathrm{~N}_{55} \mathrm{O}_{61} \mathrm{P}_{11}$ & 3952.3 & 3953.9 \\
\hline
\end{tabular}

${ }^{a}$ MALDI data were calibrated by two internal standards, T8 ([TTT TTT TT $\left.-\mathrm{H}^{-1}=2370.6\right)$ and T17 ([TTT TTT TTT TTT TTT TT $\left.-\mathrm{H}]^{-1}=5108.4\right)$.

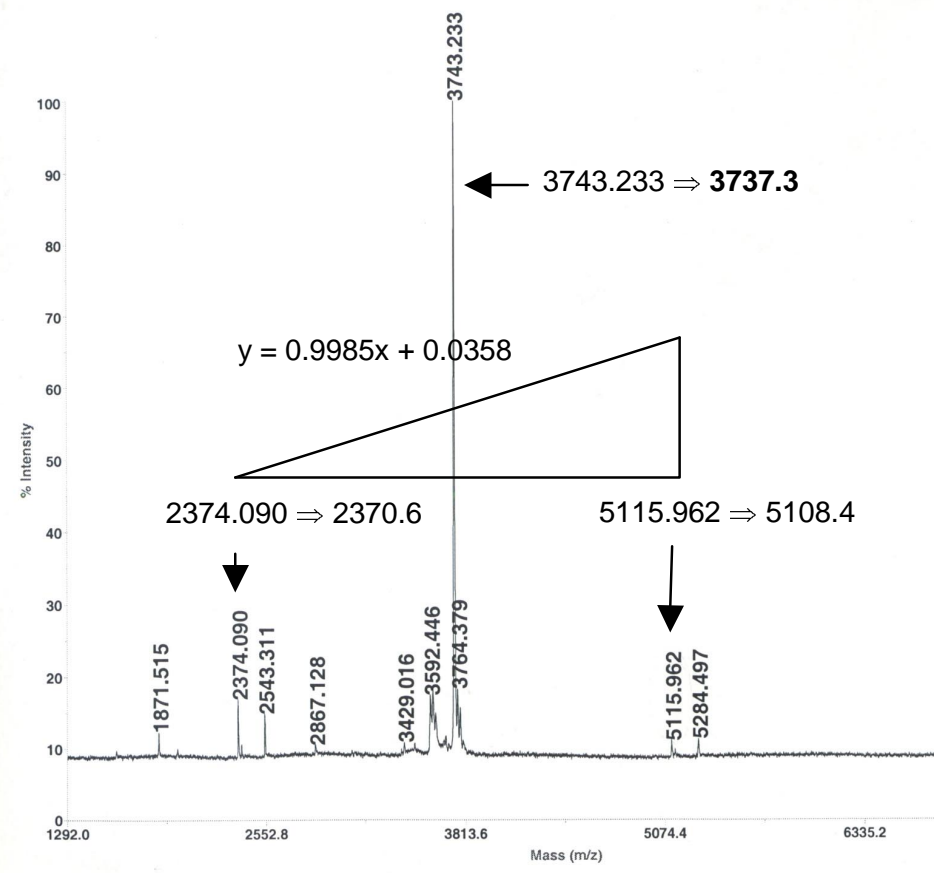

An example: MALDI MS of 3'-GAAAAGFAAGAA $\left(2^{\prime}, 3^{\prime}, 4^{\prime}\right.$-Trihyroxyacetophenone matrix, ammonium hydrogencitrate co-matrix) and its calibration.

B) Stacking Oligomers with Nonnatural Dangling End (5'-XCGCGCG) ${ }^{\mathrm{a}}$

\begin{tabular}{clcc}
\hline Sequence & & calcd MS & obsd MS \\
\hline 5'-HCGCGCG & $\mathrm{C}_{69} \mathrm{H}_{88} \mathrm{~N}_{24} \mathrm{O}_{39} \mathrm{P}_{6}$ & 2062.4 & 2062.9 \\
5'-FCGCGCG & $\mathrm{C}_{69} \mathrm{H}_{86} \mathrm{~F}_{2} \mathrm{~N}_{24} \mathrm{O}_{39} \mathrm{P}_{6}$ & 2098.4 & 2098.5 \\
5'-LCGCGCG & $\mathrm{C}_{69} \mathrm{H}_{86} \mathrm{Cl}_{2} \mathrm{~N}_{24} \mathrm{O}_{39} \mathrm{P}_{6}$ & 2131.3 & 2131.3 \\
5'-BCGCGCG & $\mathrm{C}_{69} \mathrm{H}_{86} \mathrm{Br}_{2} \mathrm{~N}_{24} \mathrm{O}_{39} \mathrm{P}_{6}$ & 2220.2 & 2220.4 \\
5'-ICGCGCG & $\mathrm{C}_{69} \mathrm{H}_{86} \mathrm{H}_{2} \mathrm{~N}_{24} \mathrm{O}_{39} \mathrm{P}_{6}$ & 2314.2 & 2314.5 \\
\hline
\end{tabular}

\footnotetext{
${ }^{a}$ MALDI data were calibrated by two internal standards, T4 $\left([\text { TTT T }-\mathrm{H}]^{-1}=1153.8\right)$ and T7 $\left([\text { TTT TTT T }-\mathrm{H}]^{-1}=\right.$ 2066.4).
} 


\section{References}

1. Fusita, T.; Iwasa, J.; Hansch, C. J. Am. Chem. Soc. 1964, 86, 5175.

2. Nowick, J. S.; Chen, J. S.; Noronha, G. J. Am. Chem. Soc. 1993, 115, 7636. 
